Original Paper http://ajol.info/index.php/ijbcs http://indexmedicus.afro.who.int

\title{
Effets des fumures organo-minérales et des rotations culturales sur la qualité des sols
}

\author{
Noufou OUANDAOGO ${ }^{1 *}$, Badiori OUATTARA ${ }^{1}$, Mathias Bouinzemwendé POUYA ${ }^{1}$, \\ Zacharia GNANKAMBARY ${ }^{2}$, Hassan Bismark NACRO ${ }^{3}$ et Papaoba Michel SEDOGO ${ }^{1}$ \\ ${ }^{1}$ Institut de l'Environnement et de Recherches Agricoles (INERA).Centre de Recherches Environnementales, \\ Agricoles et de Formation de Kamboinsé. O1B.P.476 BF-Ouagadougou, Burkina Faso. \\ ${ }^{2}$ Bureau National des Sols (BUNASOLS). 03 B.P. 7142 BF-Ouagadougou 03, Burkina Faso. \\ ${ }^{3}$ Université Polytechnique de Bobo Dioulasso (UPB) Institut du Développement Rural (IDR) BP 1091 Bobo- \\ Dioulasso, Burkina Faso. \\ *Auteur correspondant ; E-mail : ouandaogo_noufou@yahoo.fr
}

\section{RESUME}

Les sols des régions semi-arides de l'Afrique de l'Ouest sont sujets à une dégradation graduelle de leur fertilité. Ils sont connus être d'une productivité très limitée et les moyens modernes pour augmenter et maintenir cette productivité sont le plus souvent très onéreux pour les petits exploitants. Ainsi, pour trouver de solutions probantes pour une gestion durable de la fertilité des sols, une étude a été conduite à la station de recherche agricole de Saria au Centre Ouest du Burkina Faso sur un dispositif implanté depuis 1960 où des fumures organique et/ou minérale combinées à des systèmes de cultures sont testés. L'objectif de cette étude est d'évaluer les effets de ces fumures combinés aux rotations culturales, sur le statut organique et la qualité des sols. Ce dispositif expérimental est du type Split splot à 6 répétitions, combinant six niveaux de fertilisation et à trois types de rotation. La monoculture de sorgho et la rotation sorgho/niébé ont été retenues dans cette étude. Des échantillons de sol ont été prélevés à $0-20 \mathrm{~cm}$ dans les parcelles du dispositif, et dans une jachère naturelle jouxtant le site expérimental et qui a servi de référence. La texture du sol et les teneurs en carbone et azote ont été déterminées. Les résultats obtenus montrent que, plus que le carbone organique total du sol, c'est plutôt sa fraction labile qui se révèle être un indicateur très sensible aux changements précoces qui affectent le statut organique des sols. En effet, l'indice de gestion du carbone qui apparait très corrélé avec la stabilité structurale du sol se positionne comme un outil fiable d'évaluation de la qualité des sols soumis aux pratiques culturales. Par ailleurs, les rotations sorgho-niébé se révèlent être des pratiques culturales les plus appropriées pour assurer une durabilité de la qualité des sols et des productions agricoles.

(C) 2016 International Formulae Group. All rights reserved.

Mots clés : Fumures minérales et organo-minérales, rotations culturales, carbone, azote, Burkina Faso.

\section{Effects of organo-mineral fertilizers and crop rotations on soil quality}

\begin{abstract}
In the semiarid tropics of west Africa, agricultural lands are gradually degrading leading to decline soil fertitily. The low soil fertility is also increasingly recognized as a fundamental cause of declining food security in small-farm households. In this region, sustainable soil fertility management is constantly a challenge for crop productions. To come with an alternative soil fertility management practices, a trial was set up in 1960 in
\end{abstract}


the agricultural research station of Saria to identify the optimal soil management strategies for specific cropping systems to ensure sustainability of productivity. The experimental design is a split plot with 6 replications, with the main plots being three rotation system and the subplots consisting to 6 fertility management options. The main objective of this study was to evaluate the effects of soil fertility management practices combining crop rotation and fertility management options under continuous sorghum cropping and sorghum-cowpea (Vigna unguiculata) rotation systems in Burkina Faso on soil organic matter status and soil quality. Soil samples were collected at 0-20 cm depth. A bush fallow (more than 60 years old) of Andropogon gayanus located near the experimental siten is consider as natural condition for accessing changes due to landuse practices. Particle-sizes distribution, soil total $\mathrm{N}$ and carbon were determinated. The results indicated that labile organic pools are more sensitive to land use than total soil organic carbon. Carbon management index (CMI) which appeared highly correlated with soil structural stability is positioned as a reliable tool for soil quality assessment under several cropping systems. Furthermore, cowpea - sorghum rotation system seem to be the most appropriate cultural practice in ensuring sustainability of soil quality and agricultural production.

(C) 2016 International Formulae Group. All rights reserved.

Keywords: Mineral fertilizer combined with organic manure, crop rotation, carbon, nitrogen, Burkina Faso.

\section{INTRODUCTION}

Les sols de l'Afrique subsaharienne se caractérisent par des niveaux de dégradation particulièrement élevés (Thombiano et Tourino-soto, 2007). Cette situation est certes dépendante des caractéristiques intrinsèques des sols tropicaux, au regard de leur faible teneurs en argiles avec une forte dominance de la kaolinite et de leur faible statut organique (Ouattara et al., 2009). Mais elle est également liée aux conditions climatiques aggravées par les pratiques culturales inadaptées (agriculture de type minier) et par la forte pression sur les ressources en sol (Powlson et al., 2011; Meshesha et al., 2012). Au Burkina Faso, cette dégradation des sols s'accompagne inexorablement d'une baisse de leur fertilité, et plus particulièrement de leur teneur en matière organique compromettant ainsi la durabilité des systèmes de productions agro-sylvo-pastorales. En effet, les sols de Burkina sont caractérisés par de faibles taux de matières organiques (inférieur à $1 \%$ ). Or celle-ci constitue non seulement, une source et un réservoir de nutriments, mais joue également un rôle fondamental dans le maintien de la fertilité des sols tropicaux (Bationo et al., 2007; Coulibaly et al., 2012). Un appauvrissement du sol en matière organique entraîne un effondrement de l'architecture des sols, réduit l'efficacité d'utilisation des fertilisants minéraux et par conséquent, entraîne une baisse de la capacité productive des sols
(Meshesha et al., 2012). La matière organique apparaît ainsi comme le pivot de la fertilité des sols tropicaux. Le maintien ou l'augmentation du stock organique dans les sols peut se faire à travers les apports d'amendements organiques et de fertilisants organo-minéraux (Heitkam et al., 2011 ; E et al., 2012), le régime du travail du sol qui conditionne largement son stockage dans le sol (Ouattara et al.,2011), ou à travers les rotations culturales à base de légumineuses (Yusuf et al., 2009). Aussi, le maintien d'un stock organique satisfaisant dans les sols cultivés est-elle une composante intégrale de la stratégie de gestion durable de ces sols au Burkina Faso.

Par ailleurs la matière organique ne constitue pas un compartiment homogène dans les sols, en terme d'emplacement, de dynamique et d'impact sur les propriétés physico-chimiques et biologiques du sol (Six et al., 2002; Ouédraogo et al., 2014a ). Ainsi, certains pools de la matière organique sont fortement protégés par la fraction argileuse du sol (Balesdent et al., 2000). D'autres, par contre, représentés par fraction libre de la taille des sables, les carbohydrates, le carbone de la biomasse microbienne, peuvent être considérés comme des indicateurs très sensibles, comparativement au carbone totale du sol, pour caractériser les changements qui affectent le statut organique des sols sous les effets induits des pratiques culturales (Xu et al., 2011). De nombreux travaux ont montré 
que l'intégration du pool organique total du sol et du carbone labile (fraction organique libre) dans la détermination de l'indice de gestion du Carbone (CMI), peut servir de paramètre très efficace pour évaluer l'évolution de la qualité des sols soumis aux pratiques culturales (Lou et al., 2011 ; Xu et al., 2011). C'est dans cette optique que le fractionnement physique de la matière organique du sol est très souvent utilisé pour déterminer l'incidence des modes de gestion de la fertilité des sols sur la dynamique d'évolution des différents pools de carbone organique des sols, et fournir les paramètres nécessaires à la détermination du CMI (Traoré et al., 2015). Selon Ding et al. (2012), l'apport combiné de matière organique et des fertilisants minéraux impactent positivement sur le carbone total et sur les différentes fractions organiques $\mathrm{du}$ sol. Une problématique d'ensemble de gestion du stock de matière organique à un niveau agronomique acceptable se pose avec acuité pour les sols du Burkina Faso. Elle est au centre de la préoccupation de cette étude qui vise à appréhender l'impact à long terme de différentes pratiques culturales (fumures et rotations culturales) sur le statut organique, et leur incidence sur la qualité des sols dans un dispositif expérimental mis en place depuis 1960 à la station de recherche agricole de Saria, au Centre-Ouest du Burkina Faso.

\section{MATERIEL ET METHODES}

\section{Site de l'étude}

L'étude a été conduite à la station de recherche agricole de Saria, au Centre Ouest du Burkina Faso (12 ${ }^{\circ} 16^{\prime}$ Nord et $2^{\circ} 9^{\prime}$ Ouest). Le dispositif expérimental a été installé depuis 1960, et régulièrement suivi. Les sols sont des lixisols (WRB, 2006). Les caractéristiques physico-chimiques de l'horizon 0-20 cm varient de 0,3 à $0,5 \mathrm{~g} \cdot \mathrm{kg}^{-1}$ pour le $\mathrm{C}$ total ; de 0,3 à $0,5 \mathrm{~g} \cdot \mathrm{kg}^{-1}$ pour le $\mathrm{N}$ total, de 7 à $12 \%$ pour l'argile, essentiellement composée de kaolinite, et de 2 à $4 \mathrm{cmol} \mathrm{(+)} \mathrm{kg}^{-1}$ pour la CEC (Kambiré, 1994). Le climat est de type nord soudanien caractérisé par une pluviosité moyenne annuelle de $750 \mathrm{~mm}$ concentrée sur 4 à 5 mois de l'année, et par une forte variation de la température.

\section{Dispositif d'expérimentation au champ}

Le dispositif expérimental est du type Split splot à 6 répétitions, combinant six niveaux de fumures minérales et organominérales à trois types de rotation (monoculture de sorgho, rotation sorgho/coton et rotation sorgho/niébé). Seules la monoculture de sorgho et la rotation sorgho/niébé ont été considérées dans cette étude.

Les six traitements principaux sont les suivants :

$\mathrm{T}_{1}$ : témoin absolu désigné (te) ;

$\mathrm{T}_{2}$ : fumure minérale faible $(\mathbf{f m r}: 37 \mathrm{~N}-23 \mathrm{P}-$ $14 \mathrm{~K}-6 \mathrm{~S}-1 \mathrm{~B}$ pour le sorgho, $21 \mathrm{~N}-35 \mathrm{P}-21 \mathrm{~K}-9 \mathrm{~B}-$ $1.5 \mathrm{~S}$ pour le cotonnier et $14 \mathrm{~N}-23 \mathrm{P}-14 \mathrm{~K}-6 \mathrm{~S}-1 \mathrm{~B}$ pour la légumineuse) + recyclage des pailles de sorgho ;

$\mathrm{T}_{3}$ : fumure minérale faible + fumier $(\mathbf{f m o}: 5 \mathrm{t}$ $\left.\mathrm{ha}^{-1} 2 \mathrm{ans}^{-1}\right)+$ exportation des pailles de sorgho ;

$\mathrm{T}_{4}$ : fumure minérale faible sans fumier, avec exportation de paille de sorgho (fm);

$\mathrm{T}_{5}$ : fumure minérale forte (FMO : $60 \mathrm{~N}-23 \mathrm{P}-$ $44 \mathrm{~K}-6 \mathrm{~S}-1 \mathrm{~B}$ pour le sorgho, 74N-46P-38K$12 \mathrm{~S}-2 \mathrm{~B}$ pour le cotonnier et, $14 \mathrm{~N}-23 \mathrm{P}-44 \mathrm{~K}-$ $6 \mathrm{~S}-1 \mathrm{~B}$ pour la légumineuse), + fumier $(40 \mathrm{t}$ $\left.\mathrm{ha}^{-1} 2 \mathrm{ans}^{-1}\right)+$ exportation des pailles de sorgho;

$\mathrm{T}_{6}$ : fumure minérale forte sans fumier, avec exportation de paille de sorgho (FM). Les parcelles sont annuellement labourées au tracteur à la profondeur de 15 à $20 \mathrm{~cm}$.

\section{Prélèvement d'échantillons de sols}

Le prélèvement des échantillons de sols a été fait avant le labour, en juin 2012. Un échantillon composite a été constitué à partir de trois (03) prélèvements élémentaires à la profondeur $0-20 \mathrm{~cm}$ en trois endroits différents dans la surface utile de chaque parcelle élémentaire. Ces échantillons ont été séchés à l'ombre puis tamisés à $2 \mathrm{~mm}$.

\section{Analyses au laboratoire \\ Fractionnement physique de la matière organique \\ L'analyse granulométrique en cinq} fractions a été réalisée selon la méthode internationale adaptée à la pipette de Robinson Khöln. Elle s'effectue sur la terre 
fine (tamisat de $2 \mathrm{~mm}$ ) après une destruction de la matière organique à l'eau oxygénée et une dispersion des argiles à l'aide de l'hexamétaphosphate de sodium (HMP).

Le fractionnement granulométrique de la matière organique a concerné les échantillons de l'année 2012 et ceux de la parcelle sous jachère depuis 1960, utilisée comme référence pour le calcul de l'indice de gestion du carbone. La méthode de fractionnement utilisée dérive de celle de Feller et al. (1983). Cinquante grammes (50 g) de sol auxquels $150 \mathrm{ml}$ d'eau distillée ont été ajoutés, ont été agités à l'aide d'un agitateur va et vient pendant deux heures, en présence de trois billes de verre de $1,5 \mathrm{~cm}$ de diamètre. La suspension ainsi obtenue a été répartie par tamisage sous eau, successivement en trois fractions granulométriques $(2 \mathrm{~mm}-200 \mu \mathrm{m} ; 200 \mu \mathrm{m}-$ $50 \mu \mathrm{m}$; et $50 \mu \mathrm{m}-20 \mu \mathrm{m})$ Les quatre fractions et le reliquat (fraction $<20 \mu \mathrm{m}$ ) ont été recueillies dans des récipients et séchées à l'étuve à $60^{\circ} \mathrm{C}$, jusqu'à l'obtention d'un poids constant. Après séchage des échantillons, la masse de sol des différentes fractions a été mesurée.

La teneur en carbone (C) a été déterminée dans les trois fractions suivant la méthode Walkley-Black (Walkley et Black, 1934). La teneur en matière organique (MO) a été obtenue par la formule suivante:

\section{MO : C x 1,72}

L'azote total été déterminé après une minéralisation préalable de l'échantillon dans un mélange $\mathrm{He}-\mathrm{H}_{2} \mathrm{SO}_{4}$. La teneur en azote total a été déterminée par colorimétrique automatique à l'aide d'un auto-analyseur (SKALAR) selon Krom (1980).

Indice de gestion du carbone

Le calcul de l'indice de gestion du carbone (CMI) pour l'année 2012 a été fait suivant la méthode Blair et al. (1995). La parcelle sous jachère permanente depuis 1960 a été utilisée comme sol de référence pour évaluer l'effet des modes de gestion de la fertilité.

où CPI est l'indice de pool de carbone et LI est l'indice de labilité. Le CPI et le LI sont calculés comme suit :
$\mathbf{C P I}=($ Carbone total $\mathrm{du}$ traitement $) /($ Carbone total du sol sous jachère).

$\mathbf{L I}=($ Labilité du carbone du traitement)/ (Labilité du carbone du sol sous jachère )

où $\mathbf{L}$ désigne la labilité du carbone et est calculée comme suit: $\mathbf{L}=($ Carbone total des fractions $2 \mathrm{~mm}-50 \mu \mathrm{m}) /($ Carbone total de la fraction $<50 \mu \mathrm{m})$.

\section{Indice de dégradation des sols}

Sur la base du taux de matière organique et de la texture du sol, la sensibilité des sols à la dégradation (St) a été estimée par la méthode proposée par Pieri (1989).

\section{$\mathrm{St}=\mathrm{MO} \times 100 / \mathrm{A}+\mathrm{Lf}$}

où $\mathrm{MO}$ est la teneur du sol en matière organique; A et Lf, les taux d'argile et de limons fins.

\section{Analyses statistiques}

Les données ont été soumises à une analyse de variance (ANOVA), avec le logiciel Genstat, version Discovery 4. La séparation des moyennes a été effectuée par le test de Student-Newman-Keuls au seuil de $5 \%$.

\section{RESULTATS \\ Variation du statut organique des sols en fonction du mode de fertilisation et de la rotation culturale \\ Les teneurs en carbone et en azote des} sols après 52 ans de culture, sont données dans le Tableau 1. Les rotations, bien que n'affectant pas significativement le statut organique des parcelles, interagissent fortement avec les fumures organo minérales. Les plus fortes teneurs en carbone et en azote totaux sont obtenues avec la fumure organominérale forte (FMO). Par contre, la fumure minérale faible avec recyclage des pailles de sorgho (fmr) ont induit de très faibles niveaux de carbone et d'azote, à l'instar du témoin (absence de fumure). Les différentes fumures ont influencé significativement $(\mathrm{p}<0,001)$ les teneurs en carbone et en azote totaux des sols (Tableau 1).

Comparativement à la jachère naturelle qui sert de référence à l'étude, les fumures et les rotations ont influencé différemment les teneurs en azote et en carbone (Tableau 1). 
Après 52 ans de culture, les teneurs en carbone ont baissé sur la parcelle n'ayant pas reçu de fertilisant et sur celles ayant été exclusivement fertilisées en engrais minéraux. Cette baisse varie entre 26 et $57 \%$ pour le carbone et de 6 à $40 \%$ pour l'azote sous culture continue de sorgho. Sous rotation sorgho/niébé, la baisse varie entre 31 et $46 \%$ pour le carbone et de 16 à $37 \%$ pour l'azote. Seules les parcelles ayant reçu du fumier (fmo et FMO) permettent de maintenir et/ou d'augmenter les teneurs en carbone et en azote, et ce, quelle que soit la rotation culturale. Les plus fortes augmentations sont observées sous la rotation sorgho-niébé, respectivement de $97 \%$ et $128 \%$ pour le carbone et l'azote.

Variation de la teneur en carbone des frations granulométriques en fonction du mode de fertilisation et de la rotation culturale

\section{Teneurs en carbone des fractions}

Le fractionnement granulométrique de la matière organique a permis d'accéder à quatre fractions qui ont été regroupées en deux groupes très contrastés (Tableau 2). Il s'agit de la matière organique particulaire de taille supérieure à $50 \mu \mathrm{m}$ et des fractions organiques peu reconnaissables à l'œil nu, de tailles inférieures à $50 \mu \mathrm{m}$. Le taux de recouvrement du carbone après fractionnement, varie de $92 \%$ à $94 \%$ sous sorgho continue et de $94 \%$ à $98 \%$ sous rotation sorgho-niébé. Les plus faibles pourcentages de perte ont été obtenus avec les sols sous jachère naturelle $(3 \%)$ et avec le traitement fmo, sous rotation sorgho/niébé.

De plus, les résultats montrent que les apports de fumures organo-minérales et les rotations culturales ont affecté, de façon significative, la fraction organique particulaire (> $50 \mu \mathrm{m}) \mathrm{du}$ sol (Tableau 2). Les parcelles ayant reçu du fumier sont celles où les plus fortes teneurs en matière organique particulaire ont été enregistrées. Les valeurs sont nettement supérieures dans le cas de la rotation sorgho/niébé, comparativement à la monoculture du sorgho $(0,63$ contre 0,54 $\mathrm{g} . \mathrm{kg}^{-1}$ )
Par contre, les fractions organiques de tailles inférieures à $50 \mu \mathrm{m}$ ne sont significativement affectées que par les seules fumures organo-minérales. Toutefois, les rotations culturales interagissent de façon très marquée avec les fumures sur ces fractions fines. Par ailleurs, elles renferment près de $80 \%$, voire plus, du carbone total et ce, quel que soit le niveau de fumure organo-minérale apportée ou le type de rotation culturale.

En considérant la jachère comme la situation de référence, les effets des différents modes de gestion de la fertilité des sols sur les teneurs en carbone des fractions organiques libres $(>50 \mu \mathrm{m})$ et des fractions organiques liées $(<50 \mu \mathrm{m})$ du sol, sont données respectivement dans les Tableaux 3 et 4 . Il ressort que les apports exclusifs d'engrais minéraux, à l'instar du témoin absolu (sans fumure), entraînent des baisses importantes des teneurs en carbone tant pour les fractions organiques libres que pour les fractions organiques liées. Mais, cette baisse est beaucoup plus accentuée sur les fractions organiques libres. Le recyclage des pailles de sorgho n'améliore guère le statut organique du sol. Seuls les apports complémentaires de fumier à faible et/ou fortes doses (fmo, FMO) parviennent à maintenir ou à améliorer le statut organique du sol. Et cette amélioration est plus accentuée sur la fraction organique libre (110 à 390\%) comparativement à la fraction organique fine (86 à 148\%). De façon globale, l'enrichissement en carbone de la fraction libre est plus élevé dans le cas de la rotation sorgho/niébé que dans la situation sous monoculture de sorgho (Tableaux 3 et 4 ).

\section{Variation de l'indice de gestion du carbone (CMI) en fonction du mode de fertilisation et de la rotation culturale}

Les indices de gestion du carbone (Tableau 5) les plus élevés ont été observés avec les traitements fertilisation organominérale à forte dose (FMO) sous rotation sorgho-niébé $(488,6)$, et fertilisation organominérale à forte dose sous culture continue de sorgho $(446,7)$. En absence de fumure organique, l'indice de gestion du carbone varie entre 26,9 et 40,8 sous culture continue de sorgho, et de 38,8 à 83,3 sous rotation 
sorgho-niébé. Les plus faibles indices de gestion du carbone ont été observés avec le témoin : 26,9 sous culture continue de sorgho, et 38,8 sous rotation sorgho-niébé. L'analyse de variance révèle des différences hautement significatives entre les différents niveaux de fumures, les rotations culturales et leurs interactions. Il ressort que seuls les traitements avec matière organique parviennent à améliorer l'indice de gestion du carbone par rapport au sol sous jachère et ce, après 52 ans de culture.

Variation de la stabilité structurale des sols en fonction du mode de fertilisation et de la rotation culturale

L'indice de sensibilité à la dégradation représente le niveau d'équilibre entre la texture fine du sol (argile et limons fins) et la matière organique $\mathrm{du}$ sol. Les résultats obtenus (Tableau 6) montrent que les apports bisannuels de 40 t.ha ${ }^{-1} \cdot 2 \mathrm{ans}^{-1}$ (FMO) ont permis d'améliorer la stabilité structurale du sol ( $\mathrm{St}>7)$ en le mettant à l'abri d'un risque de déstructuration. Par contre, les apports plus faibles (fmo : 5 t.ha ${ }^{-1} \cdot 2 \mathrm{ans}^{-1}$ ) parviennent juste à maintenir la stabilité structurale du sol à un niveau acceptable. Les sols des autres traitements sont tous physiquement dégradés et très sensibles à l'érosion $(\mathrm{St}<5)$. L'analyse de variance révèle des différences hautement significatives entre les différents niveaux de fumures $(\mathrm{P}<0,001)$, mais pas entre les rotations $(\mathrm{P}=0,420)$. Une corrélation positive et significative $\left(\mathrm{R}^{2}=0,9515\right)$ entre l'indice de sensibilité des sols à la dégradation et l'indice de gestion du carbone a été obtenue.

Tableau 1: Variation des teneurs en carbone organique et en azote total $\left(\mathrm{g}_{\mathrm{kg}} \mathrm{kg}^{-1}\right)$ en fonction du mode de fertilisation et de la rotation culturale.

\begin{tabular}{llllll}
\hline Rotation & Trait. & \multicolumn{2}{c}{ Carbone } & $\begin{array}{r}\text { N- } \\
\text { total }\end{array}$ \\
\hline & & ${\mathrm{g} \cdot \mathrm{kg}^{-1}}^{-1}$ & $\%$ & $\mathrm{~g}^{-1}$ & $\%$ \\
\hline & Jachère & 3,48 & 100 & 0,30 & 100 \\
& te & $1,49^{\mathrm{a}}$ & 42,8 & $0,18^{\mathrm{a}}$ & 60,3 \\
& $\mathrm{fmr}$ & $2,14^{\mathrm{b}}$ & 61,5 & $0,19^{\mathrm{a}}$ & 63,6 \\
& fmo & $3,54^{\mathrm{d}}$ & 101,7 & $0,37^{\mathrm{c}}$ & 123,9 \\
& fm & $2,57^{\mathrm{c}}$ & 73,8 & $0,28^{\mathrm{b}}$ & 93,7 \\
& FMO & $6,46^{\mathrm{e}}$ & 185,5 & $0,61^{\mathrm{d}}$ & 204,4 \\
& FM & $2,52^{\mathrm{c}}$ & 72,4 & $0,21^{\mathrm{a}}$ & 70,3 \\
\hline \multirow{3}{*}{ Sorgho-Niébé } & Jachère & 3,48 & 100 & 0,30 & 100 \\
& Te & $1,88^{\mathrm{a}}$ & 54,0 & $0,19^{\mathrm{a}}$ & 63,6 \\
& fmr & $2,22^{\mathrm{b}}$ & 63,8 & $0,20^{\mathrm{a}}$ & 67,0 \\
& fmo & $3,07^{\mathrm{c}}$ & 88,2 & $0,31^{\mathrm{d}}$ & 103,8 \\
& fm & $2,41^{\mathrm{b}}$ & 69,2 & $0,25^{\mathrm{c}}$ & 83,7 \\
& FMO & $6,86^{\mathrm{d}}$ & 197,0 & $0,68^{\mathrm{e}}$ & 227,7 \\
& FM & $2,55^{\mathrm{b}}$ & 73,2 & $0,22^{\mathrm{ab}}$ & 73,7 \\
\hline Traitement & F-p value & $<0,001$ & & $<0,001$ & \\
Rotation & F-p value & 0,34 & & 0,89 & \\
Trait*Rot & F-p value & $<0,001$ & & 0,003 & \\
\hline
\end{tabular}

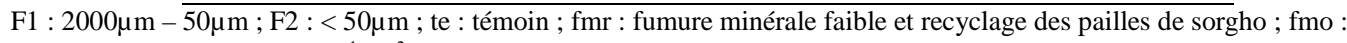
fumure minérale faible $+5 \mathrm{t} \cdot \mathrm{ha}^{-1} \cdot \mathrm{an}^{-2} ; \mathrm{fm}$ : fumure minérale faible ; FMO : Fumure minérale forte $+40 \mathrm{t} ; \mathbf{F M}$ : fumure minérale forte. Les moyennes d'une même colonne affectées de la même lettre ne sont pas significativement différentes au seuil probabilité de $5 \%$. 
Tableau 2: Variation des teneurs en carbone organique des fractions granulométriques du sol (g.kg1) en fonction du mode de fertilisation et de la rotation culturale.

\begin{tabular}{llcccccc}
\hline Rotation & & te & fmr & fmo & fm & FMO & FM \\
\hline Sorgho-Sorgho & F1 & $0,14 \mathrm{a}$ & $0,29 \mathrm{~b}$ & $0,65 \mathrm{c}$ & $0,20 \mathrm{~b}$ & $1,85 \mathrm{~d}$ & $0,22 \mathrm{~b}$ \\
& $\mathrm{~F} 2$ & $1,15 \mathrm{a}$ & $1,79 \mathrm{~b}$ & $2,65 \mathrm{~d}$ & $2,23 \mathrm{c}$ & $4,23 \mathrm{e}$ & $2,12 \mathrm{c}$ \\
Sorgho-Niébé & $\mathrm{F} 1$ & $0,20 \mathrm{a}$ & $0,29 \mathrm{~b}$ & $0,57 \mathrm{bc}$ & $0,26 \mathrm{~b}$ & $2,03 \mathrm{~d}$ & $0,41 \mathrm{bc}$ \\
& $\mathrm{F} 2$ & $1,56 \mathrm{a}$ & $1,81 \mathrm{ab}$ & $2,46 \mathrm{c}$ & $1,99 \mathrm{ab}$ & $4,52 \mathrm{~d}$ & $1,99 \mathrm{ab}$ \\
& Traitement & $(\mathrm{P}<0,001)$ & & & & & \\
Probabilité (F1) & Rotation & $(\mathrm{P}<0,001)$ & & & & & \\
& Rotation*Trait. & $(\mathrm{P}<0,001)$ & & & & & \\
& Traitement & $(\mathrm{P}<0,001)$ & & & & & \\
Probabilité (F2) & Rotation & $(\mathrm{P}<0,495)$ & & & & & \\
& Rotation*Trait. & $(\mathrm{P}<0,001)$ & & & & & \\
\hline
\end{tabular}

$\mathrm{F} 1: 2000 \mu \mathrm{m}-50 \mu \mathrm{m} ; \mathrm{F} 2:<50 \mu \mathrm{m} ;$ te : témoin ; fmr : fumure minérale faible et recyclage des pailles de sorgho ; fmo : fumure minérale faible +5 t.ha ${ }^{-1} \cdot \mathrm{an}^{-2} ; \mathrm{fm}$ : fumure minérale faible $; \mathrm{FMO}:$ Fumure minérale forte $+40 \mathrm{t} ; \mathbf{F M}$ : fumure minérale forte. Les moyennes d'une même ligne affectées de la même lettre ne sont pas significativement différentes au seuil probabilité de $5 \%$.

Tableau 3: Variation des teneurs en carbone des fractions organiques libres comparativement à la situation sous jachère naturelle.

\begin{tabular}{lcccc}
\hline Rotations/ & \multicolumn{2}{c}{ Sorgho/sorgho } & \multicolumn{2}{c}{ Sorgho/niébé } \\
\cline { 2 - 5 } Fumures & $\mathbf{g . k g}^{-1}$ & $\mathbf{\%}$ & $\mathbf{g . k g}^{-1}$ & $\mathbf{\%}$ \\
\hline Jachère & 0,52 & 100 & 0,52 & 100 \\
Te & 0,14 & 27 & 0,20 & 38 \\
fmr & 0,29 & 56 & 0,29 & 56 \\
fmo & 0,65 & 125 & 0,57 & 110 \\
fm & 0,20 & 38 & 0,26 & 50 \\
FMO & 1,85 & 356 & 2,03 & 390 \\
FM & 0,22 & 42 & 0,41 & 79 \\
\hline
\end{tabular}

te : témoin ; fmr : fumure minérale faible et recyclage des pailles de sorgho ; fmo : fumure minérale faible+ 5 t.ha ${ }^{-1} \cdot \mathrm{an}^{-2} ; \mathbf{f m}$ : fumure minérale faible ; FMO : Fumure minérale forte + 40t ; FM : fumure minérale forte. 
Tableau 4: Variation des teneurs en carbone des fractions organo-minérales comparativement à la situation sous jachère naturelle.

\begin{tabular}{lcccc}
\hline Rotations/ & \multicolumn{2}{c}{ Sorgho/sorgho } & \multicolumn{2}{c}{ Sorgho/niébé } \\
\cline { 2 - 5 } Fumures & $\mathbf{g . k g}^{-\mathbf{1}}$ & $\mathbf{\%}$ & $\mathbf{g . k g}^{-\mathbf{1}}$ & $\mathbf{\%}$ \\
\hline Jachère & 2,87 & 100 & 2,87 & 100 \\
te & 1,15 & 40 & 1,56 & 55 \\
fmr & 1,79 & 62 & 1,81 & 63 \\
fmo & 2,65 & 92 & 2,46 & 86 \\
Fm & 2,23 & 78 & 1,99 & 70 \\
FMO & 4,23 & 148 & 4,52 & 159 \\
FM & 2,12 & 74 & 1,99 & 70 \\
\hline
\end{tabular}

te : témoin ; fmr : fumure minérale faible et recyclage des pailles de sorgho ; fmo : fumure minérale faible +5 t.ha ${ }^{-1} \cdot \mathrm{an}^{-2} ; \mathbf{f m}$ : fumure minérale faible ; FMO : Fumure minérale forte $+40 \mathrm{t} ; \mathbf{F M}$ : fumure minérale forte

Tableau 5: Variation de la stabilité structurale des sols en fonction du mode de fertilisation et de la rotation culturale.

\begin{tabular}{|c|c|c|c|c|c|c|c|}
\hline Rotation & & te & fmr & Fmo & fm & FMO & FM \\
\hline Sorgho-Sorgho & & $26,9^{a}$ & $40,2^{b}$ & $136,4^{\mathrm{c}}$ & $36,5^{\mathrm{ab}}$ & $446,7^{\mathrm{d}}$ & $40.8^{b}$ \\
\hline Sorgho-Niébé & & $38,8^{\mathrm{a}}$ & $56,5^{\mathrm{b}}$ & $113,9^{\mathrm{c}}$ & $50,1^{\mathrm{b}}$ & $488,6^{\mathrm{d}}$ & $83,3^{\mathrm{c}}$ \\
\hline Probabilité & $\begin{array}{l}\text { Traitement } \\
\text { Rotation } \\
\text { Rotation*Trait. }\end{array}$ & $\begin{array}{l}(\mathrm{P}<0,001) \\
(\mathrm{P}<0,001) \\
(\mathrm{P}<0,001)\end{array}$ & & & & & \\
\hline
\end{tabular}

Te : témoin ; fmr : fumure minérale faible et recyclage des pailles de sorgho ; fmo : fumure minérale faible+ 5 t.ha $^{-1} \cdot \mathrm{an}^{-2} ; \mathbf{f m}$ : fumure minérale faible ; FMO : Fumure minérale forte $+40 \mathrm{t} ; \mathbf{F M}$ : fumure minérale forte. Les moyennes d'une même ligne affectées de la même lettre ne sont pas significativement différentes au seuil probabilité de 5\%.

Tableau 6: Variation de l'indice de dégradation des sols en fonction du mode de fertilisation et de la rotation culturale.

\begin{tabular}{lcccccc}
\hline & \multicolumn{7}{c}{ Traitement } \\
\hline Rotation & Te & fmr & fmo & fm & FMO & FM \\
\hline Sorgho-Sorgho & $2,2^{\mathrm{a}}$ & $2,4^{\mathrm{a}}$ & $4,4^{\mathrm{b}}$ & $2,2^{\mathrm{a}}$ & $10,6^{\mathrm{c}}$ & $2,8^{\mathrm{a}}$ \\
Sorgho-Niébé & $2,2^{\mathrm{a}}$ & $2,9^{\mathrm{a}}$ & $4,8^{\mathrm{b}}$ & $2,6^{\mathrm{a}}$ & $9,8^{\mathrm{c}}$ & $2,6^{\mathrm{a}}$ \\
Traitement & $(\mathrm{P}<0,001)$ & & & & & \\
Rotation & $(\mathrm{P}=0,420)$ & & & & & \\
Traitement*rotation & $(\mathrm{p}=0,051)$ & & & & & \\
\hline
\end{tabular}

te : témoin ; fmr : fumure minérale faible et recyclage des pailles de sorgho $; \mathbf{f m o}:$ fumure minérale faible+ 5 t.ha ${ }^{-1} \cdot \mathrm{an}^{-2} ; \mathbf{f m}$ : fumure minérale faible ; FMO : Fumure minérale forte $+40 \mathrm{t} ; \mathbf{F M}$ : fumure minérale forte. Les moyennes d'une même ligne affectées de la même lettre ne sont pas significativement différentes au seuil probabilité de 5\%. 


\section{DISCUSSION}

Effets des modes de gestion de la fertilité sur le statut organique des sols

Les apports de fumures organominérales et les rotations culturales ont significativement affecté à différents niveaux, les teneurs en azote et en carbone du sol. Après 52 ans de culture, on observe dans les parcelles témoin sans apports de fertilisant, ainsi que dans celles qui ont reçu des fumures exclusivement minérales, des baisses de carbone organique de 26 à $57 \%$ par rapport à la jachère naturelle qui a servi de référence. Ces résultats corroborent ceux obtenus par Mando et al. (2005) et Lompo (2009) qui ont montré que l'exploitation à long terme des sols sans apport de matière organique entraîne une baisse considérable des teneurs en carbone des sols. Du fait des conditions tropicales et de l'intensité culturale favorables à la minéralisation du carbone, cette baisse devient exponentielle au cours des premières années de cultures pour ensuite se stabiliser à un niveau minimum, comme c'est le cas sur les parcelles témoins (Katyal et al., 2001). Toutefois, l'existence de différences significatives entre le statut organique des parcelles sous fertilisation exclusivement minérale (fm, FM) et entre ces parcelles et le témoin, serait vraisemblablement due aux rhizodépositions (biomasses racinaires, biomasses microbiennes, etc.).

Les fumures organo-minérales à faible (fmo) et forte dose (FMO) ont permis de maintenir et d'augmenter les teneurs en carbone et en azote sous monoculture de sorgho, et sous rotation sorgho-niébé. En effet, de nombreux auteurs ont montré que le maintien ou l'augmentation du stock organique du sol peut se faire par le biais des fertilisations organo-minérale (Heitkam, et al., 2011 ; E et al., 2012). Les résultats obtenus confirment ceux de Pouya et al. (2013) qui ont montré que les apports bisannuels de $5 \mathrm{t} \mathrm{ha}^{-1}$ de fumier complétés avec la fertilisation minérale faible (fm) permet d'entretenir la fertilité des sols sablo-limoneux du Burkina Faso. Tout apport organique supérieur à cette dose, à l'instar de FMO, ne pourrait que contribuer à l'amélioration des propriétés physicochimiques et biologiques des sols, et donc à l'amélioration de façon durable de leur capacité productive.

L'introduction du niébé dans les systèmes de rotations n'a pas amélioré de façon significative le stock de carbone organique totale des sols. Ces résultats sont similaires à ceux obtenus par Lemke et al., (2007). Il est certes connu que dans les rotations culturales, les légumineuses contribuent à l'enrichissement du sol en azote. Les résultats obtenus sur l'azote total ne permettent pas d'affirmer clairement cette assertion, comparativement à la monoculture de sorgho. Il est toutefois à observer que les présents résultats ont été obtenus à partir de prélèvements de sol réalisés après la culture de niébé. Or cette culture, au regard de ses besoins en nutriments, n'a reçu que $14 \mathrm{~kg} \cdot \mathrm{ha}^{-1}$ d'azote contre 37 et $60 \mathrm{~kg} \cdot \mathrm{ha}^{-1}$ pour le sorgho, respectivement en doses faible (fm) et forte (FM). Malgré les écarts observés dans les doses d'azote apportées, les rotations sorgho/niébé ont permis de maintenir le statut azoté des parcelles à un niveau proche, voire supérieur à celui des parcelles sous monoculture de sorgho quel que soit le traitement.

La restitution des pailles de sorgho (fmr) n'a pas entraîné à long terme, une augmentation significative des teneurs en carbone des sols. Xu et al. (2011) ont obtenu les mêmes résultats, et ont lié cela d'une part à l'insuffisance de la quantité des substrats organiques apportés, et d'autre part au fait que les substrats apportés ont été fortement exposés à une minéralisation rapide, entraînant par conséquent un faible stockage du carbone dans le sol.

\section{Effet sur le carbone des fractions granulométrique du sol}

Le taux de recouvrement maximal du carbone issus du fractionnement physique de 
la matière organique du sol a été de 94 et 98 $\%$, respectivement sous la monoculture de sorgho et sous la rotation sorgho/niébé. Ces valeurs sont conformes à celles généralement données dans la littérature (Segda, 2006). Les pertes en carbone sont plus faibles dans les jachères naturelles car elles renfermaient deux (2) fois plus de polysaccharides (composés organiques très polymérisés et très peu hydrosolubles) que dans les champs (Sedogo et al., 1994). .

Les fumures organo minérales et les rotations culturales ont significativement affecté les teneurs en carbone des fractions libres $(>50 \mu \mathrm{m})$ et liées $(<50 \mu \mathrm{m})$. Les apports exclusifs d'engrais minéraux ont entrainé une baisse des teneurs en carbone de ces deux compartiments granulométriques du sol. Mais cette baisse est beaucoup plus accentuée sur la fraction particulaire du sol (> $50 \mu \mathrm{m})$ qui alimente le «compartiment d'entrée » de la matière organique (Mando et al., 2004). Ce résultat est en accord avec ceux d'Awale et al. (2013), Plaza-Bonilla et al. (2014), qui ont montré que le carbone contenu dans les fractions grossières est très sensible aux pratiques culturales. En effet, ce carbone plus exposé aux microorganismes subit une minéralisation rapide comparativement à la fraction argilo-limoneuse $(0-20 \mu \mathrm{m})$ moins accessible aux microorganismes (PlazaBonilla et al., 2014). Mando et al. (2004) a défini cette fraction fine, comme étant le «compartiment de stockage » de la matière organique du sol. Cependant les teneurs en carbone de ce compartiment peuvent être affectées à la baisse, du fait de l'intensité des pratiques culturales, comme c'est le cas dans cette étude. Cela corrobore les travaux de Ouattara et al. (2009), Culman et al. (2012) qui rapportent que les labours annuels peuvent désagréger la structure du sol et exposer les matières organiques à la minéralisation.

La présente étude a permis d'apprécier le rôle des rotations céréale-légumineuse dans l'enrichissement en carbone des fractions libres $\quad(50 \mu-2000 \mu \mathrm{m})$. Ceci serait certainement dû au carbone issu de la rhizodéposition (nodules, racines du niébé, biomasse microbienne). Cet enrichissement pourrait aussi être lié à la saturation en carbone de la fraction argilo-limoneuse $(\varnothing<$ $20 \mu \mathrm{m})$. En effet, selon Chung et al. (2008) et Gulde et al. (2008), la fraction minérale du sol peut être saturée en carbone, et tout apport additionnel en carbone dû aux pratiques culturales enrichirait plutôt la fraction intermédiaire du sol.

\section{Effet des systèmes de culture sur le CMI et la stabilité structurale du sol St}

L'indice de gestion du carbone (CMI), basé sur le fractionnement physique de la matière organique, est considéré comme un outil sensible pour évaluer la capacité des systèmes de culture à améliorer la qualité des sols agricoles (Vieira et al., 2007 ; Datta et al., 2010). En se référant à la jachère naturelle, les résultats montrent que les CMI calculés évoluent étroitement avec les différents modes de gestion de la fertilité des sols. Les valeurs de CMI sont relativement faibles avec le témoin, les apports répétés d'engrais (fm, FM) et l'engrais + paille (fmr). Les apports à long terme des fumures organo-minérales ont permis d'améliorer le CMI. L'indice de gestion du carbone étant un indicateur de labilité du carbone conforte les recommandations sur la nécessité de l'apport au sol de matière organique de qualité comme le fumier ou le compost. La comparaison des effets induits par le témoin et les apports continus d'engrais minéraux au sol d'une part; et d'autre part ces derniers et les fumures organo-minérales sur le CMI montrent l'importance de la fertilisation surtout l'apport de substrats organique de très bonne qualité au sol. En effet, les fertilisations exclusivement minérales induiraient une augmentation de la biomasse racinaire, avec comme conséquence une augmentation des teneurs en carbone des fractions grossières 
(Mosddeggi et al., 2009). Mais ce sont les apports de fumier à faible et/ou forte dose (fmo, FMO) qui améliorent véritablement le CMI. Le carbone contenu dans les fractions grossières est très sensible aux pratiques culturales et plus exposé aux actions des microorganismes. Par contre, le carbone apporté par le fumier est incorporé par complexation à la fraction argilo-limoneuse moins accessible aux microorganismes. Il est le pourvoyeur du carbone labile dans les sols (Vieira et al., 2007 ; Datta, 2010). En outre, c'est ce qui justifie en partie, les valeurs élevées des indices de dégradation des sols des parcelles sous apports de fumures organominérales qui présentent un moindre risque de dégradation.

L'indice de gestion du carbone est plus élevé sous rotation sorgho-niébé. Ce qui confirme le rôle et l'importance de cette pratique culturale dans la gestion durable de la fertilité des sols. Vieira et al. (2007) ont enregistré un accroissement des valeurs du CMI sous rotations culturales incluant une légumineuse, mettant ainsi en évidence le rôle des légumineuses dans l'apport additionnel au sol de carbone photosynthétisé. Ainsi, les rotations céréale-légumineuse peuvent jouer un rôle clé dans la séquestration du carbone dans les sols, et assurer par conséquent la durabilité des agrosystèmes sahéliens. Il est intéressant de noter que l'apport de matière organique, quelle que soit la dose, est indispensable pour une meilleure gestion du carbone. Ce résultat met en évidence son rôle dans la gestion durable des sols agricoles.

A défaut de procéder à une quantification au laboratoire de la stabilité structurale des sols sous les effets induits par des pratiques culturales, Piéri (1989) a défini un indice de sensibilité des sols à la dégradation (St) qui représente le niveau d'équilibre entre la texture fine du sol (argile et limons fins) et la matière organique du sol. Les apports de fumures organo-minérales faible à forte dose (fmo et FMO) ont induit une sensibilité à la dégradation du sol (St) significativement supérieure au témoin (te) sans apport de fertilisant et les fumures exclusivement minérales (fmr, fm, et FM). Ces résultats corroborent nos assertions sur l'indice de gestion du carbone (CMI) en termes de qualité des matières organiques apportés au sol. D'où la nécessité d'apporter de la matière organique de très bonne qualité comme le fumier ou le compost pour une gestion durable des sols. Au regard des résultats, il apparaît que seule la fumure organo-minérale forte (FMO) permet d'améliorer la stabilité structurale du sol (St > 7), tandis que la fertilisation organo-minérale faible (fmo) la maintient à la limite du niveau de dégradation. Ce résultat confirme une fois de plus la justesse de la recommandation de 5 t/ha de fumure organique (fmo), comme fumure dite d'entretien de la fertilité des sols (Lompo, 2009; Koulibaly et al., 2010; Pouya, 2014).

Par ailleurs, l'établissement d'une corrélation très étroite entre l'indice de gestion du carbone et (CMI) celui de la stabilité structurale (St) traduit les effets bénéfiques des flux d'énergie et de carbone sur la structure du sol (Vieira et al., 2007 ; Plaza-Bonilla, 2014). Le carbone organique et les agrégats structuraux interagissent mutuellement. En même temps que la matière organique humifiée stabilise les microagrégats et que la matière organique particulaire stabilise les macro-agrégats, ces mêmes entités structurales assurent une protection physique de la matière organique contre les attaques biologiques et permettent ainsi son accumulation dans le sol (Bayer et al., 2002 ; Diekow et al., 2005; Ouédraogo et al., 2014b). Au regard de ces résultats, il semble raisonnable de confirmer que l'indice de gestion du carbone se positionne comme étant un indicateur solide d'évaluation de la performance des pratiques culturales de la qualité des sols. 


\section{Conclusion}

Cette étude a servi de cadre de base pour évaluer les effets de différents niveaux de fumures organo-minérales combinés à des rotations culturales, sur le statut organique et sur la qualité des sols. Les différents modes de gestion de la fertilité des sols notamment l'utilisation des fumures minérales et/ou organiques entraînent une modification des propriétés physico-chimiques et biologiques du sol. En se référant à la jachère naturelle, les résultats obtenus montrent que plus que le carbone organique total du sol, c'est plutôt sa fraction labile qui se révèle être un indicateur très sensible aux changements précoces qui affectent le statut organique des sols. En effet, l'indice de gestion du carbone qui apparaît très corrélé avec la stabilité structurale du sol se positionne comme un outil fiable d'évaluation de la qualité des sols soumis aux pratiques culturales. Par ailleurs, Il est possible de conclure, au regard des résultats obtenus, que les rotations sorgho-niébé sont des pratiques culturales les plus appropriées pour assurer une durabilité de qualité des sols et des productions durables.

\section{CONFLIT D'INTÉRÊT}

Tous les auteurs déclarent qu'ils n'ont pas de conflit d'intérêt.

\section{CONTRIBUTIONS DES AUTEURS}

NO est l'investigateur principal; MBP a analysé les données statistiques; ZG a participé au choix du dispositif et analyses statistiques ; BO, HBN et PMS ont participé à l'encadrement scientifique et correction du dit manuscrit.

\section{REFERENCES}

Awale R, Chatterjee A, Franzen D. 2013. Tillage and N-fertilizer influences on selected organic carbon fractions in a North Dakota silty clay soil. Soil and Tillage Research, 134: 213-222. http://dx.doi.org/10.1016/j.still.2013.08.0 06

Balesdent J, Chenu C and Balabane M. 2000. Relationship of soil organic matter dynamics to physical protection and tillage. Soil and Tillage Research, 53: 215-230. http://prodinra.inra.fr/record/ 62532

Bationo A, Kihara J, Vanlauwe B, Waswa B, Kimetu J. 2007. Soil organic carbon dynamics, functions and management in West African agro-ecosystems. Agricultural Systems, 94: 1325.doi:10.1016/j.agsy.2005.08.011

Bayer C, Mielniczuck J, Martin-Neto L, Ernani PR. 2002. Stocks and humification degree of organic matter fractions as affected by no-tillage on subtropical soil. Plant Soil, 238: 133-140. doi : 10.1023/A:1014284329618

Blair GJ, Lefroy RDB, Lisle L. 1995. Soil Carbon Fractions Based on their Degree of Oxidation, and the Development of a Carbon Management Index for Agricultural Systems. Australian Journal of Agricultural Research, 46: 1459-1466. doi:10.1071/AR9951459

Chung H, Grove JH, Six J. 2008. Indications For soil carbon saturation in a temperate agroecosystem. Soil Science Society of America Journal, 72: 1132-1139. http://dx.doi.org/10.2136/sssaj2007.0265

Coulibaly K, Vall E, Autfray P, Nacro HB, Sedogo PM. 2012. Effetsde la culture permanente coton-maïs sur l'évolution d'indicateurs de fertilité des sols del'Ouest du Burkina Faso. Int. J. Biol. Chem. Sci., 6(3): 1069-1080.

Culman SW, Snapp SS, Freeman MA, Schipanski ME, Beniston J, Lal R, Drinkwater LE, Franzluebbers AJ, Glover JD, Grandy AS, Lee J, Six J, Maul JE, Mirksy SB, Spargo JT, Wander MM. 2012. Permanganate oxidizable carbon reflects a processed soil fraction that is sensitive to management. Soil Science 
Society of America Journal, 76: 494-504. doi:10.2136/sssaj2011.0286

Datta SP, Rattan RK, Chandra S .2010. Labile soil carbon, soil fertility, and crop productivity as influenced by manure and mineral fertilizers in tropics. J. Plant Nutr. Soil Sci., 173: 715-726. doi: 10.1002/jpln.200900010

Ding X, Han X, Liang Y, Qiao Y, Li L, Li N 2012. Changes in organic carbon pools after 10 years of continuous manuring combined with chemical fertilizer in a mollisol in China. Soil Till. Res., 122: 3641. doi:10.1016/j.still.2012.02.002

E, SZ, Li, XG, Chen, ZM, Li, XH, Song, JR, and Guggenberger, G 2012. Long-term fertilization and manuring effects on physically separated soil organic-matter pools under continuous wheat cropping at a rainfed semiarid site in China. J. Plant Sci., 175: 689-697. doi: 10.1002/jpln.201100215

Feller C, Bernard-Reversat F, Garcia JL, Pantier JJ, Roussos S, Van Vliet-Lanoe B.1983. Etude de la matière organique de différentes fractions granulométriques d'un sol sableux tropical. Effet d'un amendement organique (compost). Cah. O.R.S.T.O.M., sér. Pédol., 10(3): 223238.

Gulde S, Chung H, Amelung W, Chang C, Six J. 2008. Soil Carbon saturation controls labile and stable carbon pool dynamics. Soil Science Society of America Journal 72: $\quad 605-612 . \quad d x . d o i . o r g / 10.2136 /$ sssaj2007.0251

Heitkamp F, Raupp J, Ludwig B. 2011. Effects of fertilizer type and rate on labile soil fractions of a sandy Cambisol-longterm and short-term dynamics. J. Plant Nutr. Soil Sci., 174: 121-127. doi: 10.1002/jpln.201000121

Kambire SH. 1994 - Systèmes de cultures paysans et productivité des sols ferrugineux lessivés du Plateau Central (Burkina Faso) : effets des restitutions organiques. Thèse de $3^{\text {ème }}$ cycle, U.C.A.D. de Dakar, 153p.

Katyal JC, Rao NH, Reddy MN, 2001. Critical aspects of organic matter management in the tropics: the example of India. Nutr. Cycl. Agroecosyst., 61: 7788. doi: .\%310.1023/A: 1013320502810

Koulibaly B, Traoré O, Dakuo D, Zombré PN, Bondé D. 2010. Effets de la valorisation des résidus de récolte sur la nutrition minérale du cotonnier et les rendements d'une rotation coton-maïs-sorgho dans l'Ouest du Burkina Faso. Int. J. Biol. Chem. Sci., 4(6): 2120-2132.

Lemke RL, Zhang Z, Campbell CA Zentner R. 2007. Can pulse crops play a role in mitigating greenhouse gases from North American agriculture? Agron. J., 99: 1719-1725.

Lompo F. 2009. Effets induits des modes de gestion de la fertilité sur les états du phosphores et solubilisation des phosphates naturels dans deux sols acides du Burkina. Thèse de doctorat en sciences naturelles. Université de Cocody. Abidjan Cote d'Ivoire, $219 \mathrm{P}+$ annexes.

Lou Y, Xu M, Wang W, Sun X, Liang C. 2011. Soil carbon fractions and management index after 20 yr of manure and fertilizer application for greenhouse vegetables. Soil Use and Management 27: 163-169. doi: 10.1111/j.1475-2743. 2010.00325.x

Mando A, Ouattara B, Sédogo MP, Stroosnijder L, Ouattara K, Brussaard L, Vanlauwe B. 2005. Long-term effect of tillage and manure application on soil organic fractions and crop performance under Sudano-Sahelian conditions. Soil Tillage Res., 80: 95-101.

Meshesha DT, Tsunekawa A, Tsubo M. 2012. Continuing land degradation: cause-effect in Ethiopia's Central Rift Valley. Land Degrad. Dev., 23: 130-143. doi : 10.1002/ldr.1061 8 . 
Ouattara B, Ouattara K, Lompo F, YaoKouamé A, Sédogo MP. 2011. De la culture itinérante à la culture permanente: impact sur le statut organique et l'agrégation d'un sol ferrique à l'Ouest du Burkina Faso. Agronomie Africaine, 23(1):1-9.

Ouattara B, Ouattara K, Sedogo MP; YaoKouamé A. 2009. Statut poral des sols cutlivés dans la zone cotonnière Ouest du Burkina Faso. Agron. Afric., 20(3): 303312.

Ouédraogo J., Ouédraogo E., Nacro H.B., 2014a. Effet de l'interaction entre des modes de gestion de fertilité et la macrofaune sur la productivité du niébé et du sorgho en zone nord soudanienne du Burkina Faso. International Journal of Biological and Chemical Sciences, 8(1): 104-114.

Ouédraogo J, Nacro HB, Ouédraogo E, Youl S, Sedogo MP. 2014b. Amélioration de la disponibilité du phosphore par la gestion de la macrofaune du sol : cas d'un lixisol en zone semi-aride du Burkina Faso. International Journal of Biological and Chemical Sciences, 8(4): 1838-1846.

Pieri C. 1989. Fertilité des Terres de Savanes. Bilan de Trente ans de Recherche et de Développement Agricole au sud $d u$ Sahara. Ministère de la Coopération et du Développement, CIRAD-IRAT, Montpellier, France ; 444p.

Plaza-Bonilla D, Jorge Alvaro-Fuentes J, Cantero-Martınez C. 2014. Identifying soil organic carbon fractions sensitive to agricultural management practices. Soil \& Tillage Research, 139: 19-22. doi: 10.1016/j.stiil.2014.01.006

Pouya MB. 2014. Investigation en milieu paysan et capitalisation des résultats de référentiels de longues durées sur les modes de gestion de la fertilité des sols dans les agro-systèmes cotonniers du Centre et de l'Ouest du Burkina- Faso. Thèse de Doctorat Unique $(\mathrm{PhD}) \mathrm{de}$
l'Université Polytechnique de Bobo (UPB), Option : Systèmes de production Végétale/Spécialité Sciences du Sol, $191 p$.

Pouya MB, Bonzi M, Gnankambary Z, KOULIBALY B, Ouédraogo I, Ouédraogo SJ, Sedogo PM. 2013a. Perception paysanne et impact agropédologique du niveau de mécanisation agricole dans les zones cotonnières Centre et Ouest du Burkina Faso. Int. J. Biol. Chem. Sci., 7(2): 489-506.

Powlson DS, Whitmore AP, Goulding KWT. 2011. Soil carbon sequestration to mitigate climate change: a critical reexamination to identify the true and the false. Review. European Journal of Soil Science, 62: 42-55. doi: 10.1111/j.13652389.2010.01338.x

Segda Z. 2006. Gestion de la fertilité du sol pour une production améliorée et durable du riz (Oryza sativa L.) au Burkina Faso. Cas de la plaine irriguée de Bagré. Thèse de Doctorat, Spécialité : Sciences Biologiques appliquées, option : Biologie, Ecologie végétales. Université de Ougagdougou/Burkina Faso, 164p.

Sedogo MP, Lompo F, Ouattara B. 1994. Le carbone et l'azote dans les différentes fractions granulométriques d'un sol ferrugineux tropical: effets de quatre types d'amendements organiques. Sciences et Techniques, 21(1): 114-124.

Sedogo MP. 1993. Evolution des sols ferrugineux lessivés sous culture/ incidence des modes de gestion sur la fertilité. Thèse de doctorat. Université de Cocody, Côte d'Ivoire, 343 p.

Six, J, Conant, RT, Paul, EA, Paustian, K., 2002. Stabilization mechanisms of soil organic matter: implications for Csaturation of soils. Plant Soil, 241: 155176. doi: 10.1023/A:1016125726789

Thiombiano L, Tourino-Soto I. 2007. Status and trends in land degradation in Africa. In: Traoré S, Ouattara K, Ilstedt $\mathrm{U}$, 
Shmidt M? Thiombiano A, Malmer A, Nyberg G., 2015. Effect of land degradation on carbon and nitrogen pools in two soil types of a semi-arid landscape in West Africa. Geoderma, 241: 330-338.

Vieira FCB, Bayer C, Zanatta JA, Dieckow J, Mielniczuk J, He ZL. 2007. Soil carbon management index based on physical fractionation of soil organic matter in an Acrisol under long-term no-till cropping systems. Soil and Tillage Research, 96: 195-204. doi: 10.1016/j.still.2007.06.007

Walkley A, Black IA. 1934. An examination method of the detjareff and a proposed modification of the chromic acid titration method. Soil Science, 37: 29-38.

World Reference Base for soil resources (WRB). 2006. A framework for international classification, correlation and communication. Ed. FAO, World Soil Resources Reports $\mathrm{N}^{\circ} 103$, Rome, 128 p.

$\mathrm{Xu} \mathrm{M}$, Lou Y, Sun X, Wang W, Baniyamuddin M, Zhao K. 2011. Soil organic carbon active fractions as early indicators for total carbon change under straw incorporation. Biology and Fertility of Soils, 47(7): 745-752. doi: 10.1007/s00374-011-0579-8

Yusuf, AA, Iwuafor ENO, Abaidoo RC, Olufajo OO, Sanginga N. 2009. Grain legume rotation benefits to maize in the northern Guinea savanna of Nigeria: fixed-nitrogen versus other rotation effects. Nutr. Cycl. Agroecosyst, 84: 129139. doi:10.5923/j.plant.20120203.08 